\title{
Tumor Biology
}

National Cancer Institute

\section{Source}

National Cancer Institute. Tumor Biology. NCI Thesaurus. Code C19323.

Biological differences between normal and cancer cells. Studies use mechanistic approaches to explore tumor cell biology and tumor prog ression at the molecular and cellular level, and to examine cell-cell and cell-matrix interactions at the macromolecular level in whole tissues. Included is the study of the pathology and biology of solid tumors and tumor-bearing animals, chimeric, transgenic, and knock-out animals. Research focused on the development of technologies to facilitate these studies is included. 